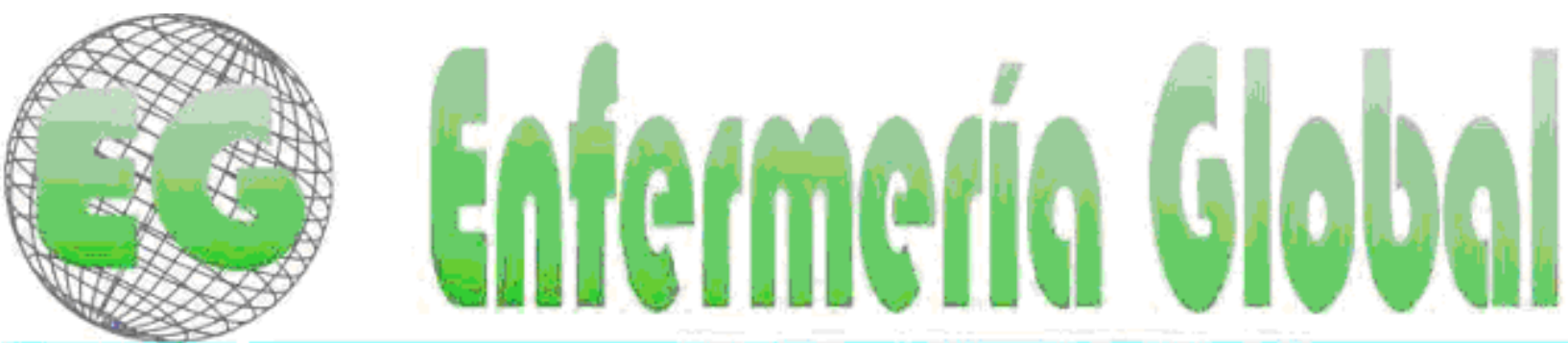

ISSN 1696-6) is:

Revista electrónica cuatrimestral de Enfermeria

$\mathrm{N}^{\circ} 18$

Febrero 2010

www.um.es/egloball

DOCENCIA - FORMACIÓN

\title{
CONFRONTACIÓN DE CRITERIOS DE EVALUACIÓN DEL APRENDIZAJE ANTE LAS APRECIACIONES DE LOS ESTUDIANTES DE ENFEMERÍA
}

\begin{abstract}
CONFRONTO DE CRITÉRIOS DE AVALIAČ̃O DA APRENDIZAGEM DIANTE DAS APRECIAÇÕES DOS ESTUDANTES DE ENFERMAGEM
\end{abstract}

\section{*Tanji, S., "Lopes Monteiro Dantas da Silva, CM dos S., "Vieira dos Santos \\ Esteves, AP., ${ }^{* *}$ Rodrigues de Oliveira, W., ${ }^{* *}$ De Paula, C.}

\begin{abstract}
*Mestre em Enfermagem. Professora do Curso de Graduação em
Enfermagem. **Especialista em Enfermagem em Emergência. Professor do Curso de Graduação em Enfermagem. Professor do Curso de Graduação em Enfermagem do Centro Universitário Serra dos Órgãos UNIFESO - Teresópolis - Rio de Janeiro.
\end{abstract}

Palabras clave: Enfermería, evaluación, proceso de enseñanza aprendizaje
Palavras chave: Enfermagem, avaliação, processo ensino aprendizagem.

Keywords: Nursing, Evaluation, Learning and Teaching Process

\section{RESUMEN}

Este estudio tiene como objetivo investigar sobre las percepciones de los estudiantes acerca de esta nueva forma de evaluación así como el impacto en su aprendizaje. El escenario del estudio fue un centro Universitario del Estado de Río de Janeiro, los sujetos fueron 26 estudiantes del tercer período en el curso de Graduación de Enfermería. La recogida de datos se realizó por medio de un instrumento con preguntas abiertas, desarrollado en el período comprendido entre abril y mayo de 2008. Los resultados fueron analizados y descritos en la unidad temática. Las tres unidades temáticas elaboradas son: el proceso de evaluación permite una evaluación fiable del conocimiento aprendizaje de los estudiantes; el proceso de evaluación permite una reflexión crítica sobre las cuestiones; la forma de evaluación requiere un mayor compromiso de los estudiantes. La percepción de los estudiantes sobre el proceso de evaluación sale al encuentro con las nuevas perspectivas de las directrices curriculares para la graduación en enfermería, especialmente en lo que se refiere a formar profesionales críticos y reflexivos. 


\section{RESUMO}

O presente estudo tem por objetivo a investigar sobre as percepções dos estudantes acerca desta nova forma avaliativa como também o impacto em seu aprender. O cenário do estudo foi um centro Universitário do Estado do Rio de Janeiro, os sujeitos foram 26 estudantes do terceiro período do curso de Graduação em Enfermagem. A coleta de dados foi realizada através de um instrumento com perguntas abertas, desenvolvido no período de abril a maio de 2008. Os resultados foram analisados e descritos em unidade temática. As três unidades temáticas elaboradas são: o processo avaliativo possibilita uma avaliação fidedigna do conhecimento - aprendizagem dos estudantes; o processo avaliativo possibilita uma reflexão crítica sobre as questões; a forma de avaliação exige mais empenho dos estudantes. As percepções dos estudantes acerca do processo avaliativo, vão de encontro com as novas perspectivas das diretrizes curriculares do curso de graduação em enfermagem, principalmente no que refere a formar profissionais críticos e reflexivos.

\section{ABSTRACT}

The objective of this study is not only to research the students' perceptions of this new assessment format but also its impact in their learning. The study took place in a University in Rio de Janeiro, Brazil; the participants were 26 students from the third part of Nursing studies. Data collection was made through an instrument with open questions carried out from April to May of 2008. Results were analyzed and described in a thematic unit. Three thematic units elaborated are: the evaluation process facilitates a reliable assessment of knowledge, the students' learning; the evaluation process facilitates a critical reflection of the questions; the assessment form requires more student effort. The students' perceptions regarding the evaluation process follow the new perspectives of the curriculum guidelines of nursing studies, mainly in regards to educating critical and reflexive professionals.

\section{CONSIDERACIONES INICIALES}

El Curso de Graduación en Enfermería del Centro Universitario Serra dos Órgãos (UNIFESO), en el primer semestre de 2007, lanza su nuevo desafío al cambio curricular. El currículo que anteriormente se estructuraba en rejas curriculares, pasa en este momento a ser constituido y elaborado de forma integrada. Esta transposición fue motivada por comprender que los currículos estructurados en rejas curriculares fragmentan los diversos saberes necesarios para el proceso de formación profesional. La opción por un nuevo currículo, orientado por competencia, se basó en el apropiar de conceptos y de metodologías diferenciadas que solamente se efectivarán en la práctica cotidiana estimuladora de cambios profundos en las relaciones vivenciadas en lo colectivo entre los actores implicados, características únicas entre los más diversos currículos en el área de la salud hasta el presente momento.

El nuevo currículo implantado en el Curso de Enfermería del UNIFESO busca la formación del enfermero comprometido con las necesidades de la población brasileña, dotado de panorama holístico e integrador, frente a los cuidados esenciales en las dimensiones individual, colectiva y planetaria.

Proponer cambios e implementarlos es un trabajo arduo, con todo otras cuestiones surgen en este contexto que nos hacen reflexionar, factor este que aún consideramos como un nudo crítico en el proceso, o sea, la evaluación del aprendizaje, para la cual ya Libâneo ${ }^{1}$, desde otrora viene presentando como una práctica llena de críticas, porque en su centro siempre incurren preceptos de cobro, control, poder, recompensa, entre tantas otras especificidades que dibujan la más pura intimidación entre los estudiantes. 
Aun así, en el siglo XXI tenemos la perfecta percepción de que las mentes se sujetan a los nuevos modelos, y que éstos deben ser ofertados a los estudiantes en el proceso de enseñanza-aprendizaje. Sin embargo, también adquirimos la conciencia de que en términos de evaluar, continuamos intimidados tanto en lo que proyectamos a los estudiantes, como lo que pretendemos con todo esfuerzo, pensar y reproducir de este modo pensamientos diferentes, en estos sujetos en formación.

Importante la reflexión porque la vivencia humana, en moldes tradicionales fue lejana y tan "perfecta" en nuestras educaciones que la postura frente a lo que nos rodea es de tal cobro y de tal exigencia que ésta remonta a los valores numéricos. Éstos representan para nosotros la real evaluación, con base en la capacidad de memorizar lo transmitido, que nos afecte en términos de capacidad y calificación, que hasta el valor "pecunia" se reviste como la mejor contraprestación, para compensar la actividad laboral que desempeñamos en nuestro trabajo como profesionales formadores de la educación del futuro. Entretanto, queda a título de reflexión, pensar que un gesto o una apreciación verbalizada de nuestra postura de profesores/tutores podría establecer la mayor diferencia, el fiel reconocimiento para proseguir adelante, innovando en el entramado de la actual educación.

La preelección es para que podamos repensar y revisar nuestros papeles ya que de evaluación de aprendizaje versará el presente texto, compuesto por mentes pensantes envueltas en las nuevas metodologías educacionales, en pro de una formación diferenciada y mejorada para nuestros estudiantes. Que se produzcan críticas, elogios y sugerencias, incluso que lo mejor oriente en este instante nuestras acciones y posturas, pero no podemos olvidarnos que la evaluación entre tantas cosas nos posibilitará siempre clarificar nuevas decisiones, valorar el estudio mismo que de nosotros emane, como un termómetro revelador de esfuerzos, en fin, para todos los actores envueltos en este nuevo proceso de aprendizaje integrador.

El término evaluar siempre causó, y aún causa, un gran temor, muchas veces por el hecho de haber sido usado el término en el sentido de medir resultados alcanzados, otras veces por los usos discriminador y punitivo que se hacen con estos resultados. No sólo en la evaluación del aprendizaje, sino también en las evaluaciones de proyectos, cursos, entre otros, donde una de las grandes preocupaciones siempre es lo que van a hacer con el resultado de la evaluación.

A pesar de todo el prejuicio existente con el término evaluar, el proceso evaluativo está presente en toda la práctica pedagógica del cotidiano del educador, aun sin claridad de constituirse evaluación.

Lo que anteriormente tuvo connotación y peso cuantitativo, hoy ¿qué cambia en este proceso? ¿Cómo debemos evaluar a nuestros estudiantes? Cuestiones éstas que mueven a intensas reflexiones a los organizadores, coordinadores del proceso de cambio

El desdoblamiento de esas exhaustivas y productivas discusiones se concretiza en un sistema de evaluación donde son valorados los aspectos cualitativos y sumativos del proceso enseñanza-aprendizaje, para que se pueda aprender a trazar junto al estudiante, lo que significa decir comprendiendo sus limitaciones y especificidades como sujeto-(a) instalado en su tiempo, ante un aprendizaje que se plantea liberador.

Muchas vueltas se han dado, para traer beneficios, siempre mejores, para los protagonistas que vivencian estos cambios y que se diga desde ya. No ha sido fácil, sin embargo, el retorno de los estudiantes más críticos y reflexivos es el mayor aliento para seguir 
introduciendo las debidas transformaciones llevándonos a constantes revisiones metodológicas, impregnándolas los arreglos de múltiples escenarios de aprendizaje. Éstos vienen desempeñando papel esencial en el perfil de los profesionales frente al formar estudiantes y los ya formados como son el tutor/facilitador, construyendo relaciones recíprocas de doble vía, en compromisos asumidos, combatiéndose las exclusiones y los autoritarismos.

Para atender a estos principios, el nuevo proceso evaluativo de los aspectos cognitivos del estudiante, que UNIFESO propone en el Curso de Graduación en Enfermería, es realizado en dos momentos distintos, que son: primer momento - evaluación continuada integrada (ECI): corresponde a un proceso de evaluación que pretende integrar las habilidades cognitivas, psicomotoras, actitudinales/afectivas adquiridas por el estudiante en los diferentes escenarios de aprendizaje. Se constituye de un sistema de evaluaciones en cada semestre, de acuerdo con el cronograma de cada curso ${ }^{2}$. Su dinámica es fundamental para la construcción del conocimiento, pues respeta el tiempo y las condiciones individuales del aprendizaje, en un método que preconiza la autonomía y el aprender a aprender en proceso.

En el segundo momento, tras la realización de la ECI escrita, el estudiante llevará consigo la Situación-Problema en cuestión y hará una búsqueda de las temáticas abordadas, construyendo una nueva síntesis manuscrita e individual con las referencias utilizadas. Esta reconstrucción hecha por el estudiante será entregada a su tutor en la sesión tutorial devolutiva. Esta sesión consiste en la presentación y discusión de estas síntesis que serán, en seguida, encaminadas al equipo que realizó la corrección de la $\mathrm{ECl}$ escrita (comisión de construcción de SP)2.

Si se detecta alguna insuficiencia en esta etapa, esta comisión encaminará al coordinador del período la prescripción individual que consiste en una recomendación de complementación o corrección de los contenidos no alcanzados y que deberá retornar a la coordinación con plazo determinado. Esta prescripción se constituye en un plan de recuperación individual, que será registrada en documento propio, visando el acompañamiento la coordinación del período. En caso de falta del estudiante en cualquier $\mathrm{ECl}$ escrita, éste debe acudir a la sesión devolutiva conforme los demás estudiantes y estará automáticamente inscrito en la $\mathrm{ECl}$ escrita de final de período, excepto en caso de Tratamiento Especial ${ }^{2}$ (situación en que el estudiante está amparado por las directrices escolares).

Por tanto el presente estudio tuvo como objetivo investigar sobre las percepciones de los estudiantes acerca de este nuevo formato evaluativo así como también el impacto en su aprendizaje.

\section{REFERENCIAL TEÓRICO}

El aprendizaje es entendido como un proceso complejo de cambio de comportamiento, englobando no solamente los aspectos cognitivos del saber, sino incluyendo también las habilidades del saber hacer y actitud de cómo saber ser y saber convivir, todos entrelazados entre sí, pudiendo utilizarse diferentes técnicas ${ }^{3}$ para la evaluación del desempeño del alumno, en el proceso de aprendizaje.

Sin embargo, es importante utilizar diferentes recursos para evaluar el desarrollo del alumno a lo largo del proceso enseñanza-aprendizaje pues en este escenario de cambios y 
dificultades estructurales, la evaluación emerge como un aspecto didáctico-pedagógico incitante y nebuloso para profesores y alumnos ${ }^{2}$.

El proceso evaluativo viene siendo ampliamente discutido en la esfera educacional, por la relevancia y por la necesidad urgente de reformular esta práctica, posibilita la toma de decisión y la mejoría de la calidad de enseñanza, informando las acciones en desarrollo y la necesidad de regulaciones constantes ${ }^{5,},{ }^{6}$. Necesita, para cumplir su verdadero significado, asumir la función de ayudar a la construcción del aprendizaje. La condición necesaria para que esto ocurra es que la evaluación deje de ser utilizada como un recurso de autoridad, que decide sobre los destinos de los estudiantes, y asuma el papel de auxiliar el crecimiento ${ }^{6}$.

Los métodos de evaluación ocupan, sin duda, espacio relevante en el conjunto de las prácticas pedagógicas aplicadas al proceso de enseñanza y aprendizaje. Evaluar, no se limita a la mecánica del concepto formal y estadístico; no es simplemente atribuir notas, obligatorias a la decisión de avance o retención en determinadas asignaturas ${ }^{5}$.

Según Perrenoud ${ }^{7}$, la evaluación del aprendizaje es un proceso mediador en la construcción del currículo y se encuentra íntimamente relacionada con la gestión del aprendizaje de los estudiantes. Para una evaluación del aprendizaje eximio, el educador no debe permitir que los resultados de las pruebas periódicas, generalmente de carácter clasificatorio, sean sobrevalorados en lugar de sus observaciones diarias, de carácter diagnóstico.

Sin embargo, con frecuencia las notas son comúnmente usadas para fundamentar necesidades de clasificación del estudiante, dentro de un continuum de posiciones, donde se da el mayor énfasis a la comparación de desempeños y no a los objetivos de la instrucción que se desea alcanzar. El estudiante es clasificado como inferior, medio o superior en cuanto a su desempeño y muchas veces quedan ligados a esos estigmas, no consiguiendo desvelar su potencial ${ }^{6}$.

El proceso de evaluación tiene la finalidad de acompañar la evolución del estudiante, identificando sus avances y dificultad, posibilitando la toma de decisiones e interviniendo cuando sea necesario ${ }^{3}$. El proceso evaluativo sin medición de notas es el más significativo para el proceso enseñanza aprendizaje, la utilización de este recurso permite al estudiante tener claro lo que es esencial y relevante del contenido en estudio, representando así un momento de reflexión sobre su caminar en busca de los desempeños esperados ${ }^{4}$.

En ese contexto, se puede decir que la evaluación es parte integrante del proceso enseñanza-aprendizaje. Requiere preparación técnica y también gran capacidad de observación de los profesionales implicados, donde la principal función de la evaluación es la diagnóstica por permitir detectar, diariamente, los puntos de conflictos generadores del fracaso escolar. Esos puntos detectados deben ser utilizados por el profesor como referenciales para los cambios en las acciones pedagógicas, objetivando un mejor desempeño del estudiante.

Como bien destaca Sacristán ${ }^{8}$, es imprescindible un esfuerzo por parte de la escuela, de sus profesionales y de sus alumnos para que no separemos los tiempos, los espacios, los modos y los sujetos - de enseñanza y de aprender. Según él, en esa separación "la evaluación se desintegra del aprendizaje, perdiendo su valor formativo en el diálogo crítico entre profesores/as y alumnos/as" 8:339.

En la evaluación del aprendizaje, el profesor no debe permitir que los resultados de las pruebas periódicas, generalmente de carácter clasificatorio, sean sobrevaloradas en 
detrimento de sus observaciones diarias, de carácter diagnóstico. El profesor, que trabaja en una dinámica interactiva, tiene noción, a lo largo de todo el semestre, de la participación y productividad de cada estudiante. Es necesario dejar claro que la $\mathrm{ECl}$ es solamente una formalidad del sistema escolar, que busca evaluar el desarrollo del estudiante y crea la posibilidad del aprendizaje en su realización.

Lo dicho anteriormente concuerda de pleno con el entendimiento traducido, sobre todo cuando en la actualidad educacional, se aprecia que el estudiante durante su formación necesita de la adquisición de competencias y habilidades, repercutiendo en una evaluación capaz de medir las transformaciones ocurridas en cada uno de estos, recordando que es preciso comprender el educar en su debida importancia de acuerdo con Delors ${ }^{9}$ :

Más que nunca la educación parece tener, como papel esencial, conferir a todos los seres humanos la libertad de pensamiento, discernimiento, sentimientos e imaginación que necesitan para desarrollar sus talentos y permanecer, tanto cuanto sea posible, dueños de su propio destino ${ }^{\text {9:100 }}$.

La evaluación del aprendizaje escolar es un medio y no fin del proceso ${ }^{6,7}$, pero debemos estar atentos a los modos de superación del autoritarismo para el establecimiento de la autonomía del estudiante. El proceso evaluativo debe manifestarse como un mecanismo de diagnóstico de la situación y tener carácter inclusivo, teniendo en cuenta el avance y el crecimiento del estudiante y no un estancamiento discisciplinador ${ }^{6}$.

Luckesi $^{6}$ destaca que la evaluación, cuando proviene del carácter clasificatorio, constituye como un instrumento estático y frenador del proceso de crecimiento, ya como proceso diagnóstico e inclusivo, ella se establece en un momento dialéctico del proceso de avanzar en el desarrollo de la acción y del crecimiento para autonomía. Perrenoud ${ }^{7}$ destaca que el educador se beneficia de una autonomía aún mayor, cuando el sistema educacional instituye una evaluación continua.

Sin embargo, la evaluación viene siendo un saber marginado en la formación, eso requiere cambio de concepción de la evaluación: el educador deja de ser el "examinador" y el estudiante, el "examinado". Se actúa en pareja, sin con eso perder el rigor y la seriedad que la actividad impone. Por el contrario, la evaluación se vuelve más exigente porque pasa a ser, también, transparente. Eso no significa retirar la responsabilidad del educador para trasladarla al educador-estudiante, pero posibilita a éste vivenciar el proceso que pueda desarrollar con sus estudiantes, de modo que sean superados los problemas que tanto hemos combatido ${ }^{10}$.

\section{MATERIAL Y MÉTODO}

Se trata de un estudio cualitativo y descriptivo, desarrollado en el período de abril a mayo de 2008, en un Centro Universitario del estado de Río de Janeiro, en el Curso de Graduación en Enfermería.

Los sujetos de la pesquisa fueron 26 estudiantes del tercer período del curso de Graduación en Enfermería. La selección de los sujetos se hizo de forma espontánea, de acuerdo con la voluntad expresa en la participación del estudio, y fueron identificados con nombre de flores, para preservación del anonimato. La recogida de datos fue realizada a través de un instrumento con preguntas abiertas. 
Destacamos que fue solicitado el previo permiso de los participantes, siguiendo las orientaciones de la Resolución №. 196/96 del Consejo Nacional de Salud, en sus principios básicos de autonomía, beneficencia, no-maleficencia y justicia, mediante el término de Consentimiento Libre y Esclarecido y tras autorización del Comité de Ética de la Pesquisa.

El análisis de los resultados contempló la saturación de los datos y después se procedió a la descripción de los mismos agrupándolos en unidades temáticas de acuerdo con la congruencia de las respuestas obtenidas, siendo estos debidamente discutidos a la luz de los referenciales teóricos que abordan la evaluación del aprendizaje.

\section{RESULTADOS Y DISCUSIONES}

He aquí llegado el momento de desvelar las perspectivas de los estudiantes concernientes a la nueva propuesta de evaluación del proceso enseñanza-aprendizaje. Cuando nos referimos a la satisfacción académica relativa a esta nueva propuesta de evaluación, todos los sujetos se manifestaron de forma positiva, siendo elaboradas tres unidades temáticas: EI proceso evaluativo posibilita una evaluación fidedigna del conocimiento - aprendizaje de los estudiantes; el proceso evaluativo posibilita una reflexión crítica sobre las cuestiones; la forma de evaluación exige más empeño de los estudiantes.

El proceso evaluativo posibilita una evaluación fidedigna del conocimiento aprendizaje de los estudiantes: esta unidad temática fue apuntada por la mayoría de los participantes del estudio, quienes creen que este formato evaluativo implementado, puede evaluar a los estudiantes de modo más justo y leal, en lo que concierne a los aspectos de su crecimiento cognitivo a lo largo del proceso formativo. Como ya hemos mencionado, la nueva propuesta de evaluación del ECl escrita, es realizada en tres momentos distintos y subsecuentes, conllevando momentos de aprendizaje en su realización, para que pueda obtener resultados cualitativos en el proceso, en estos términos Demo ${ }^{11}$ dice: la evaluación tiene como objetivo servir como instrumentalización para rehacer la ruta de inclusión del alumno, garantizándole el derecho al desempeño cualitativo satisfactorio.

No obstante, para profundizar en esta reflexión - discusión destacamos a Perrenoud ${ }^{7}$ que dice: la evaluación no es en principio, un objetivo en sí, sino un medio de verificar si los estudiantes adquirieron los conocimientos trabajados, exceptuando que la evaluación no es comparable a una simple medida o margen de error, resulta de una transacción que se basa en un nexo del desempeño escolar.

Seleccionamos algunas hablas, que compusieron esta unidad temática:

"[...] es una forma de evaluar mejor al estudiante." Lirio

"[...] cada alumno consigue saber hasta donde está alcanzando su conocimiento." Érica

"[...] puede poner lo que entendió realmente durante todas las situaciones problemas [...]" Dalia

"[...] pues en ella podremos exponer una buena parte de nuestro conocimiento libremente". Bromelia

“[...] pues en ella pude expresar mi aprendizaje". Fresáis 


\section{"[...] podremos ser evaluados más profundamente". Camelia}

El resultado del nuevo proceso evaluativo se expresa en los parámetros de suficiencia e insuficiencia, y no de forma numérica, en estos términos evocamos a Esteban ${ }^{12}$ y Luckesi $^{6}$ cuando dicen: los procesos evaluativos que sobrevaloran las notas como elementos fundamentales para evaluar los resultados del aprendizaje, son vistos como un proceso excluyente que fomenta la distancia entre el proceso y producto. En la visión crítica, la complejidad del conocimiento no se contrasta numéricamente, la evaluación debe tener como foco la valoración cualitativa del sujeto, y que sea un proceso continuum. En este contexto, Villas Boas ${ }^{10: 48}$ destaca que, "evaluar significa trabajar con las relaciones desarrolladas entre los sujetos participantes del proceso".

Luckesi $^{13}$ destaca que la evaluación es una forma de ayudar al aprendizaje satisfactorio del estudiante, a través de su acompañamiento riguroso, teniendo en cuenta su desarrollo. Añade, Marin et $a^{14: 75}$ que evaluación es una actividad permanente y dinámica del proceso enseñanza aprendizaje, con la finalidad de visualizar los avances y dificultades, lo que posibilita ajustes en el proceso. Propone acompañar y recuperar las lagunas que eventualmente puedan contener.

El proceso evaluativo posibilita una reflexión crítica sobre las cuestiones: La presente unidad temática citada por la minoría, es sin embargo de extrema relevancia, pues ante esta exposición podremos expresar que el proceso generador de las reflexiones y críticas se dispara a través de las situaciones problemas que son formuladas, y a partir de estas situaciones problemas, los estudiantes deben leer, interpretar, elaborar las hipótesis sobre el enunciado y posteriormente la construcción de las cuestiones del aprendizaje que la situación problema está encaminando, para construir las cuestiones del aprendizaje que deben desarrollarse en la evaluación. Los estudiantes deben hacer su lectura de la evaluación con ojo crítico y reflexivo, para poder desarrollar las cuestiones de forma eficaz. En este contexto, Perrenoud ${ }^{7}$ presenta algunas competencias estratégicas adquiridas por la práctica de evaluación: saber orientar las cuestiones, saber analizar sobre la importancia de la evaluación y sus dificultades, saber extraer lo esencial de una instrucción; esas habilidades exigen habilidades estratégicas e intelectuales de los estudiantes. Como dice Demo 11:92, "no queremos que el alumno memorice, decore, imite, reproduzca, sino que sepa pensar, aprenda a aprender, para poder mejor intervenir. O sea, pensar bien, para intervenir mejor". Se debe construir en torno de cada estudiante el conocimiento crítico y reflexivo, necesario y profundo, para dar cuenta del espectro de sus dificultades, avances, superaciones de su aprendizaje.

Entretanto, resaltamos que este formato evaluativo está en confluencia con las directrices curriculares del curso de graduación en enfermería, en la expectativa de formar profesionales críticos y reflexivos, con competencia política para transformar la realidad social. La propuesta de cambio incluye la formación de profesionales, suficientemente críticos y reflexivos, para que puedan actuar en el fortalecimiento del modelo de atención a la salud "usuario-centrado", donde el compromiso fundamental es con las necesidades del usuario, como contrapartida del modelo actualmente predominante "procedimientocentrado". Esto es, un modelo en el que el principal compromiso del acto de asistir a la salud es con la producción de procedimientos. Para ello, el perfil esperado del profesional de salud comprende el compromiso con la universalidad, la equidad y la integración del cuidado. La idea es que la formación debe permitir el entendimiento de la necesidad de garantía del cuidado que las personas demandan, en todas sus dimensiones, de las actividades de promoción y prevención hasta aquellas que abarcan servicios con mayor densidad tecnológica. En fin, lo que se desea es una formación que garantice el equilibrio entre la 
excelencia técnica y la relevancia social ${ }^{15}$. Siguen abajo las hablas de los participantes del estudio:

"Excelente, porque uno prepara las cuestiones de la forma con que puede contestar" Clavel

“[...] en las formulaciones de las cuestiones”. Orquídea

"[...] estimula al estudiante a pensar, reflejar y mostrar sus conocimientos". Cactos

"[...] es interpretar el texto y hacer las cuestiones del aprendizaje”. Tulipán

En estos términos apunta Vasconcelos, citado por Maia ${ }^{16}$, quien dice: La evaluación es un proceso complejo que implica una reflexión crítica sobre la práctica educativa, en el sentido de captar sus avances, sus resistencias, sus dificultades y posibilitar la toma de decisiones para superar los obstáculos.

La nueva propuesta de evaluación exige más empeño de los estudiantes: esta modalidad del proceso evaluativo moviliza a los estudiantes a ir en busca de su aprendizaje, para poder desvelar el conocimiento cognitivo que los estudiantes adquirieron durante el proceso de formación. Como dice Perrenoud ${ }^{7}$, toda la acción educativa solamente puede estimular el auto-desarrollo, el auto-aprendizaje, la auto-regulación de un sujeto, modificando su medio. Ante esto seleccionamos algunas hablas que representaron esta unidad temática:

“[...] forma más fácil, pero exige más de los alumnos”. Violeta

“[...] el alumno que tiene que esforzarse para aprender". Hibisco

“[...] los académicos están esforzándose más”. Gerbera

Pero, proponer cambios en la práctica de evaluación, para un análisis cualitativo del aprendizaje del estudiante significa mucho más que abandonar algunas técnicas e instrumentos de evaluación, implica redefinir en una dimensión teórico-práctica, el resignificar de los valores, el aprender a aprender, y por consecuencia los presupuestos de un hacer pedagógico significativo, donde el conocimiento no sea visto como algo a ser adquirido y sí como inacabado, que es el resultado de muchas experiencias de la humanidad $^{17}$.

La propuesta es desarrollar el aprender a conocer, defendido por Delors ${ }^{9}$, que es indisociable del aprender a hacer. Se considera, en la constitución de ese eje, el horizonte formativo como un proceso a través del cual el aprendizaje se torna dinámico constitutivo de la vida afuera. La formación es entendida, entonces, como proceso. No empieza ni acaba, está siempre en proceso. Es una dinámica profunda que va más allá del manejo de información ${ }^{8}$.

Así, percibimos a través de las palabras del autor la fuerte presencia de que se reviste la nueva metodología, aquí reflejada, de transformar los sujetos de un modo diferente, diría liberador, ya que dejando de estar sujetos por la reflexión y la crítica, se apoderan de bienes mayores, como habilidades, actitudes y de los cuatro pilares de la educación a la luz de Delors $^{9}$, que en todo momento hay interacción en el aprender a aprender. 
Por fin, notamos que las manos se entrelazan entre los sujetos que median la enseñanzaaprendizaje, entendiendo que lo que se aprende no está acabado y listo, como si a título de certificados, encerrase con llave de oro un futuro con el cual se obtuvieran modelos de excelencia, solo perdurasen firmados en una evaluación digna correcta que haga de sus actores seres que no crean que si algo no es evaluado, no despierta la atención, y que es en su esfuerzo de aprendizaje donde verdaderamente se evalúan los límites humanos.

La propuesta lanzada nos hace seres renovados y distintos, y los estudiantes adquieren un aprendizaje que los conforta haciendo del acto de estudiar no una presión asfixiante sino conduciéndolos como futuros profesionales, propiciando a cada instante los cambios personales, ocurridos a lo largo del proceso de aprendizaje, pues los errores mediados entre aciertos elucidan el aprender, siempre que tengamos la capacidad y la humildad de extraer de estos, los mejores y más significativos beneficios.

\section{CONSIDERACIONES FINALES}

Una evaluación se presenta con múltiples funciones y por tal motivo comporta indagaciones que nos colocan cara a diversos posicionamientos. La vivencia el estudiante, el profesortutor/facilitador, la institución, la familia, y por fin, el grupo social. Estos actores construyen un aprendizaje libre y comprometido que enaltece el verdadero aprender a aprender donde la evaluación es la única herramienta, que al ser coherente en sí misma se vuelve justa, correcta y actual.

A través de la historia de las instituciones de enseñanza se diseñaron modelos de evaluación en los que evaluar era medir, apreciar un ideal, confrontar lo bueno y lo malo, pasar o reprobar como si el universo humano fuese el resultado estático de objetividad, física y cuántica. Hoy, nos debatimos con los percances de la evaluación y nos encontramos con concepciones retrógradas las cuales concebían la validez del conocimiento que se transmitía en términos de juicio sin derecho a revisión. El evaluar provenía de métodos tradicionales de enseñanza-aprendizaje que se diseñaban de acuerdo con la autoridad y poder de un profesor que se situaba ante un estudiante pacífico y acogedor.

Las espinas dorsales de la evaluación de hoy, se comprometen con metodologías innovadoras, de tal modo que nos llevan a comprender que formar profesionales comprometidos no se hace controlando y masificando en sistemas rígidos y ciegos. En la actualidad se tiende a un aprendizaje sustentado en el diálogo, en la reflexión crítica y esencialmente científica, evaluándose a los estudiantes con todo el mérito dirigidos por una:

\section{Enfermería ética, responsable y dignamente valorizada.}

\section{REFERÊNCIAS}

1- LIBÂNEO, J. C. Didática. 25a ed. São Paulo: Cortez; 2006.

2- COA - UNIFESO (CADERNO DE ORIENTAÇÃO ACADÊMICA), 2008 [on line] Disponível em: URL: http://www.feso.br/mkt/enfermagem.pdf.

3- SAKAI MH, TAKAHASHI OC, KIKUCHI EM, ITO K. O sentido do processo de avaliação nas metodologias ativas de aprendizagem. Revista Olho Mágico 2001; 8(1): 5-7. 4- GUARIENTE, MHD de M; BERBEL, NAN; RODRIGUES, IG; UTYAMA, IKA; SOUBHIA, Z; DORNELLAS, P. Avaliação: uma questão sempre atual. Revista Olho Mágico 2001; 8(1):3436. 
5- KRAEMER, MEP. Avaliação da aprendizagem como processo construtivo de um novo fazer; 2007 [citado em: 02 de maio de 2007]. Disponível em: URL: http://www.gestiopolis.com/Canales4/ger/avaliacao.htm>

6- LUCKESI, CC. Avaliação da aprendizagem escolar: estudos e proposições. 10ª edi São Paulo: Cortez; 2000.

7- PERRENOUD, P. Avaliação: da Excelência à regulação das aprendizagens entre duas lógicas. Porto Alegre: Artmed Editora; 1999.

8- SACRISTÁN, J. Gimeno. Avaliação do ensino. In: Compreender e Transformar o Ensino. Trad. Ernani F. da Fonseca Rosa. 4aㅡ ed. Porto Alegre: Artes Médicas; 1998.

9- DELORS, J. Educação: um tesouro a descobrir - relatório para a Unesco da Comissão Internacional sobre Educação para o Séc. XXI. 10aㅡ ed. São Paulo: Cortez; 2006.

10- VILLAS BOAS, BM de F. Portfólio, Avaliação e Trabalho Pedagógico. 3ª ed. Campinas: Papirus; 2004.

11- DEMO, P. Avaliação sob olhar propedêutico. São Paulo: Papirus Editora; 1999.

12- ESTEBAN, MT. Pedagogia de projetos: entrelaçando o ensinar, o aprender e o avaliar à democratização do cotidiano escolar. In. SILVA, JF; HOFFMANN, J; ESTEBAN, MT. Organizadores. Práticas avaliativas e aprendizagem significativa. 3ํㅡㄹ Edção. Porto Alegre: Editora Mediação, 2004.

13- LUCKESI, CC. Considerações gerais sobre avaliação no cotidiano escolar. Entrevista concedida à Aprender a Fazer. Curitiba (PR) publicada em IP - Impressão Pedagógica, publicação da Editora Gráfica Expoente 2004; 36: 4-6. [citado em 05 de novembro de 2005]. Disponível em: URL: http://www.luckesi.com.br/artigosavaliacao.htm

14- MARIN, MJS; VILELA, EM; CARDOSO, CP; BRACCIALLI, LAD; PAVELQUEIRES, S; DADALTI, MRM. Fazendo e aprendendo: uma experiência de ensino/aprendizagem. Revista Brasileira de Enfermagem 2004; 57(1):75-78.

15- ALBUQUERQUE VS, TANJI S, SILVA, CMSLMD, MOÇO ETM, FELIPPE KC, MIRANDA JFA. Integração curricular na formação superior em saúde: Refletindo sobre o processo de mudança nos Cursos do UNIFESO. Revista Brasileira de Educação Médica 2007; 31(3): 296-303.

16- MAIA, ACB. Avaliação diagnóstica em educação especial: processo de integração ou exclusão. In RAPHAEL, HS; CARRARA, K. Organizadores. Avaliação sob exame. Campinas: Autores Associados; 2002.

17- LUIS, SMB. De que avaliação precisamos em arte e educação física. In. SILVA, JF; HOFFMANN, J; ESTEBAN, MT. (Org) Práticas avaliativas e aprendizagens significativas: em diferentes áreas do currículo. $3^{\underline{a}}$ ed. Porto Alegre: Editora Mediação; 2004.

18- DEMO P. Formação permanente e tecnologias educacionais. Petrópolis: Vozes; 2006.

ISSN 1695-6141

@) COPYRIGHT Servicio de Publicaciones - Universidad de Murcia 